\title{
Barreras que afectan la movilidad en bicicleta de mujeres
}

\author{
Jenny Andrea Romero González \\ Universidad Iberoamericana de México, México \\ jennyandrearomerogonzalez@gmail.com / \\ a2147295@correo.uia.mx
}

\section{Resumen}

Tramas

La movilidad se vivencia de forma singular dependiendo de múltiples elementos como la clase, la etnia, la edad, el género, entre otras. Las mujeres y los hombres tienen experiencias de movilidad diferenciadas, lo que hace que los motivos, los patrones, las responsabilidades, la autonomía y los medios de transporte que suelen usar sean distintos. Las mujeres son quienes menos utilizan la bicicleta como medio de transporte, puesto que enfrentan mayores barreras individuales, ambientales y sociales en su uso. Este ensayo plasma algunas de las barreras con las que estas se enfrentan al usar la bicicleta, así como sus posibles causas y algunas estrategias para afrontarlas. Se debe considerar el género en las acciones, programas e investigaciones que se realicen sobre la bicicleta, favorecer que las mujeres participen en la construcción de políticas públicas de movilidad y reconocer sus necesidades en el espacio público.

\section{Palabras clave}

1| bicicleta $2 \mid$ movilidad urbana $3 \mid$ barreras $4 \mid$ mujeres $5 \mid$ género

\section{Cita sugerida}

Romero González, Jenny Andrea (2021). Barreras que afectan la movilidad en bicicleta de mujeres. Tramas y Redes, (1), 143-159, 107a. DOI: 10.54871/cl4c107a 
Tramas

y Redes

Dic. 2021

$\mathrm{N}^{\circ} 1$

ISSN en trámite

\title{
Barreiras que afetam a mobilidade feminina por bicicleta
}

\begin{abstract}
Resumo
A mobilidade é vivenciada de uma forma única, dependendo de vários elementos, como classe, etnia, idade, gênero, entre outros. Mulheres e homens têm experiências diferentes de mobilidade, o que significa que os motivos, padrões, responsabilidades, autonomia e meios de transporte que costumam utilizar são diferentes. As mulheres são as que menos usam a bicicleta como meio de transporte, pois enfrentam maiores barreiras individuais, ambientais e sociais em seu uso. Este ensaio captura algumas das barreiras que eles enfrentam ao usar a bicicleta, como também suas possivieis causas e algumas estratégias para enfrentá-las. O gênero deve ser considerado nas ações, programas e pesquisas realizadas sobre a bicicleta, incentivando que as mulheres participem da construção de políticas públicas de mobilidade e reconhecendo suas necessidades no espaço público.
\end{abstract}

\section{Palavras chave}

1| bicicleta $2 \mid$ mobilidade urbana $3 \mid$ barreiras $4 \mid$ mulheres $\mathbf{5} \mid$ gênero

\section{Barriers affecting women's bicycle mobility}

\begin{abstract}
Mobility is experienced in a unique way depending on multiple elements such as class, ethnicity, age, gender, among others. Women and men have different experiences of mobility, which means that the motives, patterns, responsibilities, autonomy and the means of transport they usually employ are different. Women use bicycles as form of transportation the least, since they face greater individual, environmental and social barriers in their use. This essay studies some of the barriers they face when riding bicycles, as well as their possible causes and some strategies to face them. Gender must be considered in the actions, programs and research carried out on bicycles, encouraging women to participate in the construction of public mobility policies and recognizing their needs in public space.
\end{abstract}

\section{Keywords}

1| bicycle 2| urban mobility $3 \mid$ barriers $\mathbf{4} \mid$ women $\mathbf{5} \mid$ gender 


\section{La importancia de la movilidad}

La movilidad es una acción cotidiana que debe ser estudiada, reflexionada e intervenida de manera constante para el beneficio de todas las personas. Esta se ha convertido en un constructo teórico, social, técnico y político esquivo, frente al cual es necesario revisar sus implicaciones de manera holística (Cresswell y Uteng, 2016). Así pues, su conceptualización y comprensión entraña variados aspectos, definiciones y enfoques, que incluyen desde las más abstractas comprensiones hasta las más aterrizadas (Adey, 2017). Una definición general de este concepto es el que proponen Figueroa y Forray (2015, p. 54) como una actividad que tiene que ver con la "organización de los tiempos y los espacios de la vida cotidiana, permitiendo a los individuos organizar sus relaciones en las diversas esferas de su vida social... [Esta] se constituye por desplazamientos que poseen cierta regularidad y un carácter de obligatoriedad". En esta definición, se vislumbra que la movilidad no es simplemente transitar de un lado al otro, sino que implica diferentes dimensiones como el tiempo, el espacio, las relaciones, los desplazamientos, etc.

Existen diferentes enfoques para abordar este concepto, cada uno de ellos le da mayor prioridad a ciertos elementos o variables. Uno de esos enfoques es el de las movilidades, en el que se plantea que no existe una sola movilidad sino múltiples movilidades, cuyas características se vinculan a diversos factores, desde los más estructurales hasta los más cotidianos y experienciales (Jirón y Gómez, 2018). Los estudios de movilidades van más allá del estudio del desplazamiento como tal, en estos los movimientos de los seres humanos se comprenden como prácticas que tienen significado (Salazar, 2016). Es decir, que la movilidad se vivencia y significa de forma singular dependiendo de múltiples elementos, como, por ejemplo, el sujeto que se moviliza, la manera en que lo hace, el tiempo que utiliza, el contexto en el que se desplaza, los motivos por los que se moviliza, entre otros.

\section{Diferencias en la movilidad de hombres y mujeres}

Jirón y Gómez (2018) indican que uno de los factores que producen experiencias de movilidad diferenciadas es el género, que en muchas ocasiones puede derivar en situaciones de desigualdad y exclusión, sobre todo cuando se cruza con otros ejes de dominación como la clase, la raza, la nacionalidad, entre otras. Los estudios sobre las diferencias de movilidad entre hombres y mujeres señalan diversas causas de esta situación, entre las más relevantes están las construcciones sociales sobre el género.

Una de las grandes diferencias que se ha encontrado tiene que ver con lo que motiva la movilidad de hombres y mujeres, puesto que estos motivos se suelen relacionar con los roles tradicionales con los que se les ha asociado: en los hombres con su rol de proveedores, en el que predominan 
los desplazamientos de trabajo, y en las mujeres con su rol asociado al hogar $\mathrm{y}$ a las labores cotidianas, en el que predominan los desplazamientos aunados con el cuidado de terceras personas, que agrupan la mayor parte de sus desplazamientos (Figueroa y Waintrub, 2015).

En esta misma línea, Criado (2020) resalta la diferencia que existe entre los patrones de movilidad que realizan hombres y mujeres, al indicar que los de los hombres tienden a ser simples y directos, mientras que los de las mujeres habitualmente son más complejos, puesto que se suelen encargar del trabajo no remunerado, que requiere encadenamiento de desplazamientos, los cuales son trayectos cortos, pero interconectados y que llegan a cubrir distancias más largas.

Jakovcevic et al. (2016), por su parte, destacan las diferencias a partir de las responsabilidades que asumen y la autonomía que tienen en la movilidad. Al respecto argumentan que los viajes de las mujeres en su mayoría están relacionados con el hogar y las actividades de soporte familiar, es decir, que su movilidad es interdependiente, depende de otros, y muchas veces deben cargar víveres o personas, lo cual limita su movilidad y reduce su autonomía; mientras que los viajes de los hombres suelen ser por razones laborales, es decir, que tienen mayor control en sus horarios y formas de desplazamiento, su movilidad es más independiente. En este sentido, Vega (2004, p. 32) indica que "mientras que el varón tiende a organizar su vida cotidiana por medio de lógicas personales, la mujer asume la responsabilidad de articular las demandas del colectivo familiar".

De acuerdo con las diferencias anteriormente mencionadas, relacionadas con los motivos, los patrones, las responsabilidades y la autonomía, se puede vislumbrar que existen relaciones inequitativas de género en lo referente a las prácticas de cuidado que llevan a inequidades en la movilidad. De esta manera, las mujeres tienen en el hogar una sobrecarga de actividades asociadas con el cuidado, que también implican una sobrecarga en la movilidad, la cual se puede complejizar aún más por la doble jornada laboral, las carencias económicas y/o si se habita en lugares con alta vulnerabilidad social.

Otro elemento diferenciador en la movilidad entre hombres y mujeres tiene que ver con el medio de transporte que utilizan. Los estudios de Criado (2020), Zucchini (2015), Palacios (2012) y el de Umaña-Barrios y San Gil (2017) muestran que las mujeres son quienes realizan más desplazamientos a pie y en transporte público, mientras que los hombres suelen desplazarse más en vehículo particular, y cuando en una familia hay un solo vehículo, es el hombre quien más accede a este. Palacios (2012) indica que se deben considerar los requerimientos de cada grupo de personas en la política pública de transporte, pues es evidente que hay diferencias de movilidad entre las y los usuarios y sus formas de desplazamiento. Sin embargo, los 
operadores de transporte consideran que no existen tales diferencias y que las necesidades de todos son universales, por ello en sus estadísticas no se suelen desglosar por sexo los resultados que se obtienen. De esta manera, las necesidades de las pasajeras no son atendidas (Loukaitou-Sideris citada por Criado, 2020). Este es un claro sesgo androcéntrico en el estudio y la intervención de la movilidad.

Con respecto a la diferencia en los medios de transporte, numerosos estudios y estadísticas de transporte muestran que las mujeres usan menos la bicicleta que los hombres (Farinola, 2015; González et al., 2014; Hérick de Sá et al., 2018; Kienteka et al., 2014) puesto que además de tener que enfrentar de manera más aguda las barreras generales que se encuentran en las ciudades para su uso, también deben enfrentar algunas barreras de orden cultural, relacionadas con estereotipos sociales. La bicicleta como medio de transporte puede favorecer las necesidades de movilidad de las mujeres, asimismo, su salud y su economía al ser un medio de bajo costo; sin embargo, al parecer las barreras han sido más fuertes que los beneficios.

\section{Barreras que enfrentan las mujeres para movilizarse en bicicleta}

Se debe aprender a ser peatón, pasajero, conductor $y$, por supuesto, ciclista. Se requiere adquirir técnicas del cuerpo para manejar la bicicleta, pero también aprender a incorporarse a los sistemas de movilidad, así como cuando se aprende a caminar o a conducir un carro por la ciudad, "hay que aprender a moverse de maneras específicas y en relación permanente con objetos y cuerpos fijos o en movimiento e interiorizar ese saber como una práctica que progresivamente se naturaliza” (Salazar, 2016, p. 57). También se debe entender que estos aprendizajes son contextuales y pueden tener variaciones de un territorio a otro.

Para usar la bicicleta se deben tener en cuenta diferentes factores, entre ellos los físicos, los del tráfico, los ambientales, los del viaje y los del o la ciclista (Sousa y Penha-Sanches, 2019). Cuando un ciclista realiza un recorrido usualmente planea y escoge una ruta, en la que de manera consciente o inconsciente tiene en cuenta los siguientes elementos: a) la existencia de ciclovías o cicloruta, es decir, la infraestructura ciclista. b) La calidad del pavimento. c) Calles sin pendientes. d) Presencia de aparcamientos en el lado derecho de la calle (estacionamiento lateral). e) Necesidad de cruzar barreras urbanas como puentes, túneles, viaductos, autopistas y ferrocarriles. f) Calles con un solo carril y el ancho del camino. g) Intersecciones con semáforos y/o señales de alto. h) Velocidad del tráfico en el carril. i) Volumen de vehículos en el carril. j) Tráfico de autobuses y camiones. k) Alumbrado público nocturno. 1) Arborización (sombra). m) Camino más rápido 
- tiempo de viaje. n) Camino más corto- distancia de viaje. o) Seguridad. p) Vías unidireccionales. q) Desigualdad en los bordes de las calles (bordillos) - Desigualdad de los canales de drenaje. r) Presencia de puntos de parada de autobús. s) Contaminación del aire (Sousa y Penha-Sanches, 2019).

Hacer uso de la bicicleta para movilizarse es una alternativa democrática de bajo costo de adquisición y de mantenimiento; es respetuosa con el medio ambiente, puesto que al no consumir combustible no se emiten gases de efecto invernadero; y es saludable para quienes la usan y para quienes disfrutan de una ciudad atmosféricamente menos congestionada y ruidosa. Sin embargo, no se pueden desconocer los aspectos negativos de su uso como el riesgo de lesiones, discapacidades y muerte a las que se está expuesto en medio del tráfico (Lopes y Machado, 2012), puesto que las y los ciclistas junto a las y los peatones son los más vulnerables en la vía. Aunados a la accidentalidad se encuentran otros limitantes como los hurtos, la contaminación, los conflictos con los otros actores viales, etc.

Con respecto a estos aspectos, se ha identificado que existen varias barreras para el uso de la bicicleta como medio de transporte. Para iniciar, abordaré algunas de las barreras socioculturales relacionan con los estereotipos y roles de género con las que se enfrentan las mujeres al hacer uso de la bicicleta, sobre todo de manera utilitaria, es decir, en trayectos cotidianos a destinos funcionales como el trabajo, el mercado, la escuela, etc., dado que al hacer uso de este medio de forma recreativa o deportiva pueden llegar a enfrentarse a otras diferentes. Un gran limitante es que algunas mujeres jamás se han subido a una bicicleta, otras no aprendieron a manejarla o tuvieron malas experiencias en su infancia con ella, lo cual las llevó a que la abandonaran completamente; sin embargo, muchas de ellas desean aprender a manejarla, pero no se sienten capaces (Palacios, 2012). Los principales motivos por los que varias mujeres no aprendieron a montar bicicleta han tenido que ver con limitaciones para acceder a ella, que no las incentivaron a usarla en edades tempranas y la inseguridad que perciben para utilizarla como medio de transporte (Santa Cleta y Antropológicas, citadas por Huerta y Gálvez, 2016). Por otra parte, algunas mujeres que saben montar bicicleta no la usan como medio de transporte porque temen circular en la vía junto a los autos o sienten que no tienen las habilidades necesarias para manejarla (Palacios, 2012).

El proceso de crianza desde el nacimiento ha estado marcado por estereotipos de género que, a nivel cultural, se van reforzando. A las mujeres, desde niñas se les ha desincentivado la realización de actividad física, sobre todo de alto impacto, puesto que se considera que dejan de ser "delicadas" o "femeninas", características que se consideran parte esencial del "ser mujer". Esto se relaciona con lo que menciona De Beauvoir (2020 [1949]) sobre que constantemente se indica que la feminidad está en peligro y se nos invita a que sigamos "siendo mujeres", a que participemos en la misteriosa 
y amenazada feminidad frente a la cual ni siquiera existe un modelo claro, pero se nos pide que nos alejemos de todo lo que la ponga en riesgo, como en este caso el uso de la bicicleta.

Con relación a lo anterior, Ferreira et al. (2018) indican que a los niños se los incentiva a practicar deportes y otras actividades físicas, mientras que a las niñas se las alienta a desarrollar actividades típicamente sedentarias en interiores. Además, una sensación de inseguridad, falta de apoyo social y una infraestructura inadecuada también pueden inducirlas a ser menos activas. Gonçalves et al. (citados por Fernandes et al., 2010) mencionan que los adolescentes, en comparación a las adolescentes, tienen más apoyo social y familiar para realizar actividades físicas y que, asimismo, ellas perciben frecuentemente más barreras personales para la participación en actividades físicas en el tiempo libre.

De esta manera, "los hombres son más propensos a practicar actividades físicas de intensidad vigorosa como deportes y ejercicio, mientras que las mujeres practican más actividades ligeras/no estructuradas como el baile y las tareas domésticas" (Fernandes et al., 2010, p. 34). Esta tendencia se mantiene a lo largo de la vida por patrones socioculturales complejos y una serie de factores sociodemográficos relacionados con el entorno que los rodea (Kirchengast y Marosi citados por Pérez et al., 2012). Lo cual no favorece el uso de la bicicleta en las mujeres, puesto que es una actividad física de mediano a alto impacto que se realiza en exteriores.

Por su parte, Kienteka et al. (2014) mencionan que las mujeres también reportan sentir menos confianza en el uso y el mantenimiento de las bicicletas que los hombres. No contar con los conocimientos y las herramientas necesarias para despinchar o ajustar algún elemento de la bicicleta durante un trayecto es una gran barrera para las mujeres, sobre todo en lugares donde no hay fácil acceso a personas que se encarguen de esos ajustes. De esta manera, no solo sienten inseguridad con respecto a sus capacidades físicas para el manejo adecuado de la bicicleta (miedo a caerse, a que se burlen de ellas o a sufrir algún accidente) (De la Paz Díaz, 2017) sino también para realizar su mantenimiento.

Kienteka et al. (2012), Kienteka y Reis (2017) y Kienteka et al. (2018) han organizado en tres grupos las barreras con las que se suelen enfrentar los y las ciclistas en la ciudad, las cuales afectan de manera diferente a hombres y mujeres, lo que se puede relacionar con las barreras socioculturales anteriormente mencionadas en función del género:

1. Ambientales - Que contemplan tanto barreras físicas como naturales.

a) Funcionalidad: limitación en los elementos de acceso para bicicletas y condiciones para desplazamientos en la 
ciudad. Carencia en la infraestructura de ciclismo (ausencia o mal estado), alto volumen de tráfico, largas distancias a los destinos, falta de estacionamientos, etc. La ausencia de infraestructura ciclística influye significativamente en que las mujeres no usen la bicicleta, puesto que suelen dudar de su capacidad para maniobrarla y perciben mayor riesgo en el espacio público.

b) Condiciones de seguridad para el uso de la bicicleta y el tráfico. Infraestructura en la que se pueden presentar situaciones de conflicto con los peatones en las aceras o situaciones adversas con los carros en la vía. Presencia de tráfico pesado.

c) Clima y temperatura: lluvia, demasiado calor o frío. Contaminación ambiental.

2. Individuales - En las que se cruzan aspectos biológicos, psicológicos, cognitivos, emocionales y la toma de decisiones. En este grupo influye el sexo, el género, el grupo de edad, entre otros. No contar con bicicleta, con indumentaria o vestuario. Miedos a ser atacadas o a la accidentalidad.

3. Sociales - Se refieren al apoyo social, por ejemplo, pedalear acompañado por otro u otra ciclista se considera una aproximación del apoyo social para usar la bicicleta. No contar con estos apoyos se convierte en una barrera. Como se mencionó previamente, el apoyo social del círculo más cercano es importante para que las mujeres realicen actividades físicas $y$, en este caso, usen la bicicleta como medio de transporte. Este apoyo se puede materializar para las mujeres en contar con acompañante para realizar los trayectos, en contar con personas que las incentiven a usar la bicicleta o en no ser juzgadas por usarla.

En estas barreras, también se encuentran las referentes a la falta de seguridad ante la criminalidad (hurtos y ataques) en entornos peligrosos. Las mujeres enfrentan "el temor a la ciudad por considerarla peligrosa ya que desde pequeñas se les dice y han vivido el acoso de la ciudad como un espacio masculino" (De La Paz Díaz, 2017, p. 123). La percepción de inseguridad que suelen tener las mujeres con respecto al espacio público influye significativamente en su movilidad, puesto que limita sus desplazamientos. Frente a esta percepción existen dos posiciones: por un lado, que esta no necesariamente coincide con la inseguridad real (Figueroa y Forray, 2014), sino que pareciera ser un miedo irracional 
infundado más complicado que el crimen en sí (LoukaitouSideris citada por Criado, 2020). Pero, por otro lado, se indica que las encuestas de criminalidad no cuentan toda la historia, puesto que no contemplan una serie de conductas sexuales amenazadoras que las mujeres enfrentan en el espacio público, que no se alcanzan a configurar en un delito, pero que en suma se constituyen en una sensación de amenaza sexual: de que están siendo observadas y están en peligro (Criado, 2020). Estas conductas intimidantes no se denuncian porque no llegan a ser un delito, de esta manera las mujeres creen que no las van a tomar en serio; muestra de ello es que ni siquiera las toman en cuenta en las estadísticas de delincuencia. Además, temen ser juzgadas y que se las culpe de las agresiones que reciben (Criado, 2020).

Teniendo en cuenta lo anterior, las mujeres tienen el doble de probabilidad de sentir miedo en el espacio público (Criado, 2020). Esta sensación de inseguridad puede hacer que no contemplen el uso de medios alternativos para su movilidad como la bicicleta, puesto que se sienten más expuestas y menos protegidas al tener que enfrentarse a transitar en espacios vandalizados, poco iluminados, con poco tránsito de personas, con muchas barreras físicas en los que es más probable que se presenten situaciones de acoso sexual u otro tipo de violencias.

Es importante reconocer estas barreras en el momento de formular políticas públicas y programas que promuevan el uso de la bicicleta, así como comprender que estas barreras impactan de manera diferente en las mujeres, puesto que ellas las experimentan con mayor intensidad. Las instituciones suelen tender al orden patriarcal, por ello la mayoría de las encargadas de la movilidad han invisibilizado a las mujeres al asumir un presunto "sujeto universal o neutral" que se moviliza, el cual termina siendo un sujeto masculino, que se mueve principalmente por cuestiones laborales. De esta manera, las necesidades de movilidad de las mujeres no son tomadas en cuenta por las instituciones, lo que precariza y vulnera su derecho a la movilidad.

La brecha de género en el ciclismo es más frecuente en contextos en los que la cultura ciclista es débil o escasa, así como "también podría estar relacionada con las preferencias de infraestructura y las normas culturales, incluida una mayor aversión al riesgo entre mujeres, estereotipos fuera del grupo y experiencias de marginación” (Hérick De Sá et al., 2018, p. 7). El número de mujeres ciclistas en Latinoamérica sigue siendo muy bajo, por lo 
que es muy importante revisar las políticas públicas de movilidad en bicicleta en la región, puesto que puede presentar sesgos sexistas o partir de un presunto sujeto universal de la movilidad, en el que no se tienen en cuenta las necesidades de las mujeres y las barreras con las que se enfrentan. Esto tiene como resultado la exclusión o una interacción limitada de las mujeres en el espacio público.

Por ello, Prati (citada por Sousa y Penha-Sanches, 2019, p. 44) menciona que existe poca incidencia femenina en el uso de la bicicleta como medio de transporte, lo cual se visibiliza en el predominio de los ciclistas masculinos en las investigaciones que se han desarrollado. De igual forma, esta baja representatividad de las mujeres en las investigaciones también se da en el diseño de políticas públicas ciclo inclusivas, esta es una forma de omitir la importancia del papel de las mujeres en la movilidad (Garrard et al., citadas por Sousa y Penha-Sanches, 2019).

\section{¿Qué hacer ante estas barreras?}

Ante la necesidad de incentivar a la población a usar medios de transporte más sostenibles, se presenta una serie de retos que se deben tener en cuenta en cualquier programa o política que pretenda promover la bicicleta: reducir la accidentalidad de ciclistas, realizar mantenimiento integral de la red existente de ciclovías, implementar o fortalecer el sistema de bicicletas públicas y reducir el hurto de bicicletas; lo cual no solo atraerá a hombres jóvenes a viajar en bicicleta sino también a mujeres y personas de todas las edades (Rosas-Satizábal y Rodríguez-Valencia, 2019).

Debido a la complejidad de las barreras que enfrentan las mujeres al usar la bicicleta como medio de transporte, se deben contemplar diferentes estrategias para hacerle frente, las cuales tengan en cuenta diversas dimensiones:

Accesibilidad: Lo inicial para enfrentar las barreras es que las mujeres puedan acceder a la bicicleta, que sus costos sean moderados al igual que su mantenimiento o que puedan acceder con facilidad a las bicicletas públicas. $\mathrm{Al}$ respecto, Kienteka et al. (2014) señalan que para fomentar el ciclismo urbano es necesario que los programas se centren más en las mujeres y adultos mayores, y que se garantice el acceso a esta modalidad de transporte disminuyendo el costo de las bicicletas y del equipo complementario.

Enseñanza y actividad física: Promover la actividad física y el uso de la bicicleta en las mujeres desde la infancia. En las mujeres jóvenes, adultas y adultas mayores que no saben montar bicicleta, se deben generar estrategias y programas de enseñanza que les ayuden a manejar su cuerpo en la bicicleta, 
a incorporarse a las dinámicas de movilidad en este medio y a combatir sus miedos.

Diseño espacial y seguridad integral: Algunas investigaciones sobre movilidad (Hérick De Sá et al., 2016; Umaña-Barrios y San Gil, 2017) han mostrado que el diseño espacial influye en la percepción de seguridad en el espacio público y, por ende, en la movilidad de las mujeres. De esta manera, el diseño de la infraestructura ciclística debe contemplar las necesidades de las mujeres y su alta percepción de riesgo. En este sentido, el Observatorio de Mujeres y Equidad de Género de Bogotá [OMEG] (2019) señala algunas de las variables que se deberían tener en cuenta en los diseños de ciclorrutas seguras para mujeres:

- Iluminación: Son necesarias ciclorrutas bien iluminadas que permitan ver con claridad el camino.

- Densidad de personas: Que exista la presencia de un volumen alto de personas cerca de la ciclorruta.

- Diversidad de personas: Que la presencia de personas sea mixta, de diferente sexo y edad.

- Presencia de agentes de seguridad: Ciclorrutas con la presencia de fuerza pública o vigilancia privada.

- Sendero: Estado de la ciclorruta o lo que la rodea, que sea un espacio en el que se pueda caminar o correr.

- Transporte público: Acceso a este medio de transporte cerca de la ciclorruta.

- Apertura o visión libre: Hace referencia a lo que se puede ver mientras se maneja, es decir, a ciclorrutas que hacen posible ver de manera clara en todas las direcciones, que permitan a la ciclista dimensionar una posible situación de riesgo.

- Visibilidad: Hace referencia a los sujetos que pueden ver a la ciclista mientras maneja, es decir, ciclorrutas en las que es probable que varias personas vean a la ciclista.

Accidentalidad y equipos de protección: Se debe disminuir la accidentalidad con la construcción de infraestructura exclusiva para las ciclistas y alentar el uso de equipos de protección (García et al., 2013), como, por ejemplo, casco, espejo retrovisor al lado izquierdo, campana, luces, prendas reflectivas, etc. Kienteka y Reis (2017) señalan que una manera de disminuir la accidentalidad es con el aumento de ciclistas en las calles, puesto que esto los hace más visibles para los conductores y peatones, además, aumenta la probabilidad de que los conductores sean usuarios de bicicletas y en consecuencia sean más conscientes sobre los derechos de las ciclistas en la vía. 
Investigación: Es importante realizar mayores investigaciones sobre el tema dado que son muy pocos los estudios en Latinoamérica sobre el uso de bicicleta en mujeres. Investigaciones de este tipo aportarán a reconocer de manera más clara las necesidades y mejorar las condiciones de las ciclistas en la ciudad. La movilidad es una actividad muy importante en la vida de las personas, por ello es necesario estar actualizando el conocimiento sobre este tema.

Género: Este debe ser considerado en las acciones, programas, planes e investigaciones que se realicen sobre el tema, junto a otros organizadores sociales como la clase, la raza, la edad, la nacionalidad, entre otras, que en el entrecruzamiento con el género pueden precarizar de diferentes maneras la movilidad en bicicleta de la diversidad de mujeres. Superar la idea del sujeto universal y neutral de la movilidad. Es importante vincular el género en las discusiones y la promoción de la movilidad sostenible, que este sea otro pretexto para seguir construyendo formas de relacionarnos más igualitarias y equitativas. Desde las instituciones, se debe favorecer la perspectiva de género en diversos ámbitos, que las mujeres participen en la construcción de políticas públicas de movilidad urbana sostenible y que se tengan en cuenta sus necesidades como usuarias del espacio público, el cual históricamente se les ha negado o limitado.

\section{Reflexiones finales a modo de conclusión}

La movilidad refleja estructuras de poder que se vuelven invisibles y mantienen dinámicas propias de la modernidad en las que se divide (por ejemplo, a través de los roles tradiciones), se jerarquiza (se le da mayor importancia a la movilidad masculina que se orienta a la producción y no a la reproducción y los cuidados) y se domina (se ubica a la diversidad de las mujeres en una posición de desventaja en la movilidad). De esta manera, las relaciones de poder en la movilidad son acordes a las que se presentan en otros ámbitos de la vida cotidiana.

La movilidad es un derecho y una acción habitual que realizan las personas, que debe ser revisada, analizada e intervenida continuamente, teniendo en cuenta los diferentes organizadores sociales, entre ellos el género. En este sentido, las mujeres y los hombres se movilizan de forma diferente, por diversas razones que se abordaron en este documento. Sin embargo, las políticas públicas de movilidad, la planeación urbana y la gestión del transporte tienden a manejar la idea de un sujeto universal y neutral de la movilidad, lo que deja de lado las necesidades específicas de las mujeres y les vulnera este derecho.

Las mujeres suelen ser principalmente peatonas y usuarias del transporte público, ante lo cual se les presentan múltiples barreras que deben 
afrontar para movilizarse de manera cotidiana. No obstante, las barreras que se les presentan en relación con otros medios de transporte son mucho mayores. Esto sucede, por ejemplo, con el uso de la bicicleta, para la utilización de la cual deben afrontar un gran número de barreras de orden individual, ambiental y, sobre todo, sociocultural, relacionadas con estereotipos de género. Ante la complejidad de estas barreras, se deben considerar diversas estrategias que abarquen diferentes dimensiones como la accesibilidad, la enseñanza, la promoción de actividad física, el diseño espacial, la seguridad integral, la reducción de accidentalidad, el uso de equipos de protección, la investigación y la perspectiva de género.

De igual forma, se debe reconocer la heterogeneidad de mujeres que se mueven en las ciudades. Por ello se deben tener en cuenta otros organizadores sociales y diferencias, aparte del género, los cuales se entrelazan en las experiencias de las mujeres, puesto que algunas pueden experimentar en el espacio público mayores opresiones y vulnerabilidad en su movilidad. Es decir, que también se requiere una mirada interseccional en lo referente a la movilidad en bicicleta.

El cuerpo de las mujeres en el espacio público sobre una bicicleta es toda una afrenta al orden patriarcal, puesto que es un lugar que presuntamente no nos pertenece y el uso de la bicicleta pone en riesgo los estereotipos de feminidad, que incluyen desde el vestuario que se usa hasta el movimiento de mediano y alto impacto que se realiza. Que las mujeres usen la bicicleta se puede configurar en una forma de lucha contra el capitalismo y el patriarcado: es una manera de cuestionar la hegemonía de la industria del carro, el cual tiene la primacía en nuestras ciudades, no solo en la vía, sino que también en espacios que usurpa que son de los peatones o ciclistas, puesto que estacionan en ocasiones en los andenes y en las ciclorrutas.

Ante este poder hegemónico aparecen las resistencias colectivas, en este caso las colectivas de ciclistas que se organizan para invitarnos a movilizarnos y apropiarnos del espacio público de otra manera con la bicicleta. Se genera un poder colectivo y creativo que promueve que las mujeres se cuestionen esos estereotipos de género que las limita y, además, sirven de apoyo social para muchas mujeres que no encuentran este apoyo en su círculo más cercano. De esta manera, ante tantas barreras, emergen también las resistencias que pretenden cuestionarlas y minimizarlas, así como, reivindicar nuestro derecho al espacio público y a una movilidad libre de violencias sexistas. 


\section{Referencias}

Adey, Peter (2017). Mobility. New York: Routledge.

Cresswell, Tim y Uteng, Tanu (2016). Gendered mobilities: towards an holistic understanding. En Gendered mobilities (pp. 15-26). New York: Routledge.

Criado, Caroline (2020). La mujer invisible: descubre cómo los datos configuran un mundo hecho por y para los hombres. Barcelona: Seix Barral.

De Beauvoir, Simone ([1949] 2020). El segundo sexo. Buenos Aires: Penguin Random House.

De la Paz Díaz, María (2017). La bicicleta en la movilidad cotidiana: experiencias de mujeres que habitan la Ciudad de México. Revista Transporte y Territorio, (16), 112-126. http://revistascientificas2. filo.uba.ar/index.php/rtt/article/view/3605

Farinola, Martín (2015). Viajes cortos, actividad física y emisiones vehiculares en la ciudad de Buenos aires. Hacia la Promoción de la Salud, 20(2), 43-58. https://revistasojs.ucaldas.edu.co/index.php/ hacialapromociondelasalud/article/view/2164

Fernandes, Rômulo; Cristofaro, Diego; Casonato, Juliano; Costa Rosa, Clara; Costa, Felipe; Freitas, Ismael; Monteiro, Henrique y Ramos, Arli (2010). Leisure time behaviors: prevalence, correlates and associations with overweight in Brazilian adults. A cross-sectional analysis. Revista Médica de Chile, (138), 29-35. https://scielo. conicyt.cl/pdf/rmc/v138n1/medica\%201-2010.pdf\#page=29

Ferreira, Rodrigo; Varela, Andrea; Zaranza, Luciana; Häfele, Cesar; Santos, Simone; Wendt, Andrea y Silva, Inácio (2018). Sociodemographic inequalities in leisure-time physical activity and active commuting to school in Brazilian adolescents: National School Health Survey. Cadernos de Saúde Pública, 34(4). https://doi. org/10.1590/0102-311X00037917

Figueroa, Cristhian y Forray, Rossana (2015). Movilidad femenina: los reveses de la utopía socio-espacial en las poblaciones de Santiago de Chile. Revista de Estudios Sociales, (54), 52-67. https://journals. openedition.org/revestudsoc/9449

Figueroa, Cristhian y Waintrub, Natan. (2015). Movilidad femenina en Santiago de Chile: reproducción de inequidades en la metrópolis, el barrio y el espacio público. Urbe. Revista Brasileira de Gestão Urbana, 7(1), 48-61. https://doi.org/10.1590/2175-3369.007.001. $\mathrm{AO} 03$

García, Leila Posenato; Freitas, Lucia Rolim Santana de y Duarte, Elisabeth Carmen (2013). Deaths of bicycle riders in Brazil: characteristics and trends during the period of 2000-2010. Revista 
Brasileira de Epidemiologia, 16, 918-929. https://doi.org/10.1590/ S1415-790X2013000400012

González, Silvia; Sarmiento, Olga; Lozano, Óscar; Ramírez, Andrea y Gri-

jalba, Carlos (2014). Niveles de actividad física de la población colombiana: desigualdades por sexo y condición socioeconómica. Biomédica (Bogotá), 34(3), 447-459. https://www.redalyc.

Hérick de Sá, Thiago; Machado, Leandro; Borges, María; Nakamura Anapolsky, Sebastián; Parra, Diana; Adami, Fernando y Monteiro, Carlos (2018). Prevalence of active transportation among adults in Latin America and the Caribbean: a systematic review of population-based studies. Revista Panamericana de Salud Pública, 41, 35 https://doi.org/10.26633/RPSP.2017.35

Hérick de Sá, Thiago; Moraes, Rafael; Duran, Ana y Monteiro, Carlos (2016). Socioeconomic and regional differences in active transportation in Brazil. Revista de Saúde Pública, 50, 37. https://doi. org/10.1590/S1518-8787.2016050006126

Huerta, Elena y Gálvez, Cristina (2016). Mujeres en bicicleta. Imaginarios, prácticas y construcción social del entorno en la ciudad de Sevilla. Antropología Experimental, (16), 111-128. https://doi. org/10.17561/rae.v0i16.3020

Jakovcevic, Adriana; Franco, Paul; Dalla, Marcela y Ledesma, Rubén (2016). Percepción de los beneficios individuales del uso de la bicicleta compartida como modo de transporte. Suma Psicológica, 23(1), 33-41 https://doi.org/10.1016/j.sumpsi.2015.11.001

Jirón, Paola y Gómez, Javiera (2018). Interdependencia, cuidado y género desde las estrategias de movilidad en la ciudad de Santiago. Tempo Social. Revista de Sociología da USP, 30(2), 55-72. https:// doi.org/10.11606/0103-2070.ts.2018.142245

Kienteka, Marilson; Rech, Cassiano y Fermino, Rogério (2012). Validade e fidedignidade de um instrumento para avaliar as barreiras para o uso de bicicleta em adultos. Revista Brasileira de Cineantropometria \& Desempenho Humano, 14(6), 624-635. https://doi. org/10.5007/1980-0037.2012v14n6p624

Kienteka, Marilson; Reis, Rodrigo Siqueira y Rech, Cassiano Ricardo (2014). Personal and behavioral factors associated with bicycling in adults from Curitiba, Parana State, Brazil. Cadernos de Saúde Pública, 30, 79-87. https://doi.org/10.1590/0102-311X00041613

Kienteka, Marilson y Reis, Rodrigo (2017). Validity and reliability of an instrument in Portuguese to assess bicycle use 
patterns in urban areas. Revista Brasileira de Cineantropometria \& Desempenho Humano, 19(1), 17-30. https://doi. org/10.5007/1980-0037.2017v19n1p17.

Kienteka, Marilson; Camargo, Edina; Fermino, Rogério y Reis, Rodrigo (2018). Quantitative and qualitative aspects of barriers to bicycle use for adults from Curitiba, Brazil. Revista Brasileira de Cineantropometria \& Desempenho Humano, 20(1), 29-42. https:// doi.org/10.5007/1980-0037.2018v20n1p29

Lopes, Mauren y Machado, Carlos (2012). Pedalando em busca de alternativas saudáveis e sustentáveis. Ciência \& Saúde Coletiva, 17(6), 1617-1628 https://doi.org/10.1590/S1413-81232012000600024

Observatorio de Mujeres y Equidad de Género de Bogotá (OMEG) (2019). Me muevo segura. Boletín Mujer-es en cifras 18. Mediciones nocturnas en materia de seguridad para las mujeres - Ciclorrutas. http://omeg.sdmujer.gov.co/index.php/mediciones/ me-muevo-segura

Palacios, Alfredo (2012). Rodada de Altura 2012. Urbano, 15(25), 67-69. https://www.redalyc.org/pdf/198/19824826009.pdf

Pérez, Betty; Marrodán, María; Aréchiga, Julieta; Prado, Consuelo y Cabañas, María (2012). Actividad física y su repercusión en la composición corporal en adolescentes venezolanos. Archivos Venezolanos de Puericultura y Pediatría, 75(4), 100-107. http:// ve.scielo.org/scielo.php?pid=S000406492012000400003\&script $=$ sci_arttext\&tlng=pt

Rosas-Satizábal, Daniel y Rodríguez-Valencia, Álvaro (2019). Factors and policies explaining the emergence of the bicycle commuter in Bogotá. Case Studies on Transport Policy, 1(7), 138-149 https:// doi.org/10.1016/j.cstp.2018.12.007

Salazar, Oscar (2016). Fervor y marginalidad de las ciclomovilidades en Colombia (1950-1970). Revista Colombiana de Sociología, 39(2), 49-67. https://doi.org/10.15446/rcs.v39n2.58965

Sousa, Isabel-Cristina Nunes de y Penha-Sanches, Suely (2019). Fatores influentes na escolha de rota dos ciclistas. EURE (Santiago), 45(134), 31-52. http://dx.doi.org/10.4067/S0250-71612019000100031

Umaña-Barrios, Nancy y San Gil, Andrea (2017). How can spatial design promote inclusivity, gender equality and overall sustainability in Costa Rica’s urban mobility system? Procedia Engineering, 198, 1018-1035. https://doi.org/10.1016/j.proeng.2017.07.147

Vega, Pablo (2004). Movilidad y vida cotidiana de mujeres de sector popular de Lima: Un análisis del testimonio de la señora Eufemia. Anthropologica, 22(22), 31-62. http://www.scielo.org.pe/scielo. php?pid=S0254-92122004000100002\&script=sci_arttext 
Zucchini, Elena (2015). Género y transporte: análisis de la movilidad del cuidado como punto de partida para construir una base de conocimiento más amplia de los patrones de movilidad. El caso de Tramas Madrid [Tesis de doctorado en Planificación Territoriall. Universidad Politécnica de Madrid, Madrid, España. http://oa.upm. Dic. 2021 es/39914/ 